\title{
Cobalt Chloride Pretreatment Promotes Cardiac Differentiation of Human Embryonic Stem Cells Under Atmospheric Oxygen Level
}

\author{
*Kwong-Man Ng, ${ }^{1-3}$ *Yau-Chi Chan, ${ }^{3}$ Yee-Ki Lee, ${ }^{3}$ Wing-Hon Lai, ${ }^{3} \mathrm{Ka}-$ Wing Au, ${ }^{3}$ Man-Lung Fung,,2 \\ Chung-Wah Siu, ${ }^{1,3}$ Ronald A. Li, ${ }^{1-4}$ and Hung-Fat Tse ${ }^{1,3,4}$
}

\begin{abstract}
Our previous study demonstrated the direct involvement of the HIF- $1 \alpha$ subunit in the promotion of cardiac differentiation of murine embryonic stem cells (ESCs). We report the use of cobalt chloride to induce HIF- $1 \alpha$ stabilization in human ESCs to promote cardiac differentiation. Treatment of undifferentiated hES2 human ESCs with $50 \mu \mathrm{M}$ cobalt chloride markedly increased protein levels of the HIF- $1 \alpha$ subunit, and was associated with increased expression of early cardiac specific transcription factors and cardiotrophic factors including NK2.5, vascular endothelial growth factor, and cardiotrophin-1. When pretreated cells were subjected to cardiac differentiation, a notable increase in the occurrence of beating embryoid bodies and sarcomeric actinin-positive cells was observed, along with increased expression of the cardiac-specific markers, MHC-A, MHC-B, and MLC2V. Electrophysiological study revealed increased atrial- and nodal-like cells in the cobalt chloride-pretreated group. Confocal calcium imaging analysis indicated that the maximum upstroke and decay velocities were significantly increased in both noncaffeine and caffeine-induced calcium transient in cardiomyocytes derived from the cobalt chloride-pretreated cells, suggesting these cells were functionally more mature. In conclusion, our study demonstrated that cobalt chloride pretreatment of hES2 human ESCs promotes cardiac differentiation and the maturation of calcium homeostasis of cardiomyocytes derived from ESCs.
\end{abstract}

\section{Introduction}

$\mathbf{H}$ UMAN EMBRYONIC STEM CELLS (ESCS), derived from the inner cell mass of blastocysts, can self-renew while maintaining their pluripotency to become all cell types including highly specialized and terminally differentiated cells such as cardiomyocytes (Moore et al., 2005; Siu et al., 2007). Successful differentiation of cardiomyocytes from human ESCs represents an important step in the development of cardiovascular regenerative medicine. The clinical application of human ESC-derived cardiomyocytes nonetheless remains questionable due to the extremely low tendency of spontaneous cardiac differentiation from cultured human ESCs as well as the immaturity of the differentiated cardiomyocytes.
Previous studies have demonstrated that low oxygen tension or hypoxia plays an important role in the proliferation, differentiation, and maintenance of the cardiovascular system during embryonic development (Fisher and Burggren, 2007; Huang et al., 2004; Ramirez-Bergeron et al., 2004; Simon and Keith, 2008; Xue and Tomanek, 1999). Although reduced oxygen tension appears to direct cultured ESCs to differentiate into cardiomyocytes (Niebruegge et al., 2009), the underlying molecular mechanism remains unclear. At a cellular level, the handling of a hypoxia signal involves the hypoxia inducible factor 1 (HIF-1), a transcription factor that consists of an oxygen labile alpha subunit (HIF-1 $\alpha$ ) and an oxygen inert beta subunit (HIF-1 $\beta$ ) (Wang et al., 1995). Our recent study demonstrated that direct increased HIF- $1 \alpha$ expression via gene

\footnotetext{
${ }^{1}$ Research Centre of Heart, Brain, Hormone and Healthy Ageing, Li Ka Shing Faculty of Medicine, University of Hong Kong, Hong Kong, Republic of China.

${ }^{2}$ Department of Physiology, University of Hong Kong, Hong Kong, Republic of China.

${ }^{3}$ Cardiology Division, Department of Medicine, Queen Mary Hospital, University of Hong Kong, Hong Kong, Republic of China.

${ }^{4}$ Stem Cell \& Regenerative Medicine Consortium, Li Ka Shing Faculty of Medicine, University of Hong Kong, Hong Kong, Republic of China.

*The first two authors contributed equally to this article.
} 
transfer promotes cardiac differentiation and maturation of cultured mouse ESCs (Ng et al., 2010). These findings suggest that HIF- $1 \alpha$ may enhance cardiac differentiation of ESCs. The therapeutic application of HIF- $1 \alpha$ gene transfer to promote cardiomyocyte differentiation of ESCs is nevertheless limited by the use of a viral vector. A virus-free method that can increase the HIF- $1 \alpha$ expression would thus be preferable.

The effects of direct reduced-oxygen treatment on cardiac differentiation of human ESCs are inconsistent and nonreproducible. Ezashi et al. (2005) showed that reduced oxygen tension prevents differentiation of human ESCs. On the other hand, Chen et al. (2009) demonstrated that 5\% oxygen culture is not beneficial for the maintenance of human ESCs in an undifferentiated state with short splitting intervals. Despite the fact that reduced oxygen treatment may enhance cardiac differentiation of ESCs (Niebruegge et al., 2009), the number of consequent viable cardiomyocytes may be limited by poor cell survival during prolonged culture with reduced oxygen tension treatment ( $\mathrm{Ng}$ et al., 2010). Other methods such as pharmacological treatment with divalent ions such as cobalt (II) and nickel (II) ions (Bunn and Poyton, 1996) to stabilize the HIF- $1 \alpha$ subunit may thus provide an attractive alternative. In fact, cobalt chloride has been used in stabilizing the HIF- $1 \alpha$ in both cell culture and animal models in various studies. For example, application of cobalt chloride has been shows to increase the HIF- $1 \alpha$ stability in acute myeloid leukemic cells (Huang et al., 2003) as well as human nasal polyp fibroblasts (Lin et al., 2008), whereas short-term cobalt administration caused HIF-1 in the kidney tubules in a rat model (Matsumoto et al., 2003). The exact reason accounted for hypoxia mimicking property of cobalt chloride remains inconclusive. Because the degradation of HIF- $1 \alpha$ requires prolyl hydroxalation and such process utilizes iron ion as cofactor, it is likely that cobalt chloride increases the HIF- $1 \alpha$ stability via its iron antagonistic action (Kaelin, 2002; Kivirikko and Myllyharju 1998).

In the present study, we sought to investigate the use of cobalt chloride to stabilize the HIF- $1 \alpha$ protein at normal oxygen levels to enhance cardiac differentiation and maturation of human ESCs. The results of this study provide new insight in the development of a novel strategy to improve the differentiation and functional property of cardiomyocytes derived from human ESCs.

\section{Materials and Methods}

\section{Maintenance and cardiac differentiation of hES2 cells}

Human ESC, the hES2 line, was obtained from the WiCell Research Institute (Madison, WI). The undifferentiated cells were maintained in a serum- and feeder-free environment using the StemPro hESC SFM system (Invitrogen, Carlsbad, CA) according to the manufacture's instruction. Where necessary, hES2 cells (at 60\% confluence) were treated with cobalt chloride (at the final concentration of $50 \mu \mathrm{M}$ ) for 3 days prior to cardiac differentiation. For cardiac differentiation, when cells reached $80 \%$ confluence, cell colonies were treated with dispase $(1 \mathrm{mg} / \mathrm{mL})$ for $5 \mathrm{~min}$ at $37^{\circ} \mathrm{C}$ and scraped off into small aggregates. The cell aggregates were allowed to become embryoid bodies (EB) by being cultured in suspension in the StemPro hESC medium for 5 days. After suspension, EBs were plated onto a gelatin-coated plate in a basal medium [StemPro34, Invitrogen, containing $2 \mathrm{mM}$ glutamine, $4 \mathrm{mM}$ monothioglycerol (MTG), $50 \mu \mathrm{g} / \mathrm{mL}$ ascorbic acid, and $0.5 \mathrm{ng} / \mathrm{mL}$ bone morphogenetic protein-4 [BMP4]) according to previous protocol (Yang et al., 2008). The following concentrations of factors were used for primitive-streak formation and for mesoderm induction for cardiac differentiation: BMP4, $10 \mathrm{ng} / \mathrm{mL}$; human basic fibroblast growth factor (bFGF), $5 \mathrm{ng}$ / $\mathrm{mL}$; activin A, $3 \mathrm{ng} \mathrm{mL}^{-1}$, and human DKK1, $150 \mathrm{ng} / \mathrm{mL}$. Factors were added as follows: days 1-4, BMP4, bFGF, and activin A; days 4-8, DKK1; after day 8, DKK1 and bFGF. The bFGF was purchased from Peprotech (Rockyhill, NJ), DKK1 from Gibco (Grand Island, NY). and activin-A from Stemgent (San Diego, CA).

\section{Western blot analysis}

The total proteins from hES2 cells were isolated as described previously (Ng et al., 2010, 2011). Fifty micrograms of total soluble protein was resolved on $10 \%$ PAGE and transferred onto a PVDF membrane electrically. After incubating in the blocking solution $(1 \times$ Tris-buffered saline, $0.05 \%$ Tween-20, 5\% nonfat powdered milk), the membrane was incubated with the primary antibody as indicated in the figure at $4^{\circ} \mathrm{C}$ for $16 \mathrm{~h}$. Unbound antibodies were removed by three washes in washing buffer $(1 \times$ Tris-buffered saline, $0.05 \%$ Tween-20), and the membrane incubated with an HRP-conjugated antimouse IgG secondary antibody (GE Healthcare, Piscataway, NJ) at room temperature for $1 \mathrm{~h}$. Free antibodies were removed by three washes in washing buffer, and the presence of the protein-antibody complex was visualized using the ECL Plus Western blotting detection reagents (GE Healthcare).

\section{End-stage PCR and real-time quantitative $P C R$ analysis}

Total RNA was isolated and reverse transcribed using the illustra RNAspin Mini Kit (GE Healthcare): $0.5 \mu \mathrm{g}$ was reverse-transcribed into first-strand cDNA using the QuantiTect Rev. Transcription Kit (Qiagen, Chatsworth, CA).

Table 1. Primers Used for RT-PCR

\begin{tabular}{|c|c|c|}
\hline Gene & Direction & Sequence (5" to $\left.3^{\prime}\right)$ \\
\hline \multirow[t]{2}{*}{ HIF-1 $\alpha$} & Forward & TGATGACCAGCAACTTGAGG \\
\hline & Reverse & GCGGAACTGCTTTCTAATGG \\
\hline \multirow[t]{2}{*}{ HIF- $2 \alpha$} & Forward & TGGATTTCGGGAATCAGAAC \\
\hline & Reverse & CTGCTGCTGAAACTTGTCCA \\
\hline \multirow[t]{2}{*}{$\mathrm{HIF}-3 \alpha$} & Forward & TGGCTATAGTCCCGATGACC \\
\hline & Reve & GGGAGTGGCTGCTACTGAAG \\
\hline \multirow[t]{2}{*}{ HIF- $1 \beta$} & Forward & CAAGCCCCTTGAGAAGTCAG \\
\hline & Reve & TCTGTCCAGTCTCAGGAGCA \\
\hline \multirow[t]{2}{*}{ HIF- $2 \alpha$} & Forward & TGAAGAAGACGCTGATGTGG \\
\hline & Rever & GGGGACCTGGGATAAGTCAT \\
\hline \multirow[t]{2}{*}{ EGLN2 } & Forward & GTACTACGGCATCTGCGTCA \\
\hline & Reve & CCGGTCAGACCAGAAAATGA \\
\hline \multirow[t]{2}{*}{ EGLN1 } & Forward & GGCCAAGGGAAAAGTAAAGG \\
\hline & Reverse & TTCCCGTTACAGTGGCGTAT \\
\hline \multirow[t]{2}{*}{ EGLN3 } & Forward & AATTGCCCTGGAGTACATCG \\
\hline & Reverse & ACTTCGTGTGGGTTCCTACG \\
\hline \multirow[t]{2}{*}{ PH-4 } & Forward & GTGACGGAGTGCTGAGTCTG \\
\hline & Reverse & CCCATCAGGCAGGTAGTTGT \\
\hline \multirow[t]{2}{*}{$\mathrm{FIH}$} & Forward & AGAATGAGGAGCCTGTGGTG \\
\hline & Reverse & GGGTGATGAACAGGGTATGG \\
\hline
\end{tabular}


Table 2. Primers Used for Real-Time QuAntitative RT-PCR

\begin{tabular}{|c|c|c|}
\hline Gene & Direction & Sequence ( $5^{\prime}$ to $\left.3^{\prime}\right)$ \\
\hline \multirow[t]{2}{*}{ HIF- $1 \alpha$} & Forward & TGCATCTCСАТСТССТАССС \\
\hline & Reverse & CGTTAGGGCTTCTTGGATGA \\
\hline \multirow[t]{2}{*}{ GATA4 } & Forward & GATCTTCGCGACAGTTCCTC \\
\hline & Reverse & CATGGCCAAGCTCTGATACA \\
\hline \multirow{2}{*}{ Nkx2.5 } & Forward & TTCCCGCCGCCCCCGCCTTCTAT \\
\hline & Reverse & CGCTCCGCGTTGTCCGCCTCTGT \\
\hline \multirow[t]{2}{*}{ VEGF } & Forward & СCTTGCTGCTCTACСТССАС \\
\hline & Reverse & CACACAGGATGGCTTGAAGA \\
\hline \multirow[t]{2}{*}{ CTF-1 } & Forward & GGAGGGAAGTCTGGAAGACC \\
\hline & Reverse & CTGCACATATTCCTGGAGCA \\
\hline \multirow[t]{2}{*}{ NGN } & Forward & AGTGACCTATCCGGCTTCC \\
\hline & Reverse & AGACGCCCGGGAGATATTG \\
\hline \multirow[t]{2}{*}{ MYOG } & Forward & GTGGGCGTGTAAGGTGTGTA \\
\hline & Reverse & AGCAGGGTGCTTCTCTTCAG \\
\hline \multirow[t]{2}{*}{ GATA1 } & Forward & ACTGCCCATCTCTACCAAGG \\
\hline & Reverse & CCACAGTAGTGCTGGTGGTG \\
\hline \multirow[t]{2}{*}{ SPI1 } & Forward & AAGAAGATCCGCCTGTACCA \\
\hline & Reverse & TTGTGCTTGGACGAGAACTG \\
\hline \multirow[t]{2}{*}{ OCT4 } & Forward & $\begin{array}{l}\text { ACATCAAAGCTCTGCAGAAA } \\
\text { GAACT }\end{array}$ \\
\hline & Reverse & $\begin{array}{l}\text { CTGAATACСТTCССАAATAGA } \\
\text { АССС }\end{array}$ \\
\hline \multirow[t]{2}{*}{ SOX2 } & Forward & AACCCCAAGATGCACAACTC \\
\hline & Reverse & GCTTAGCCTCGTCGATGAAC \\
\hline \multirow[t]{2}{*}{ NANOG } & Forward & $\begin{array}{l}\text { CAGCTGTGTGTACTCAATGA } \\
\text { TAGATTT }\end{array}$ \\
\hline & Reverse & $\begin{array}{l}\text { CAACTGGCCGAAGAATAGCA } \\
\text { ATGGTGT }\end{array}$ \\
\hline \multirow[t]{2}{*}{ MHC-A } & Forward & GATGCCCAGATGGCTGACTT \\
\hline & Reverse & GGTCAGCATGGCCATGTCCT \\
\hline \multirow[t]{2}{*}{ MHC-B } & Forward & $\begin{array}{l}\text { TGCAAAGGCTCCAGGTCTGA } \\
\text { GGGC }\end{array}$ \\
\hline & Reverse & $\begin{array}{l}\text { GCCAACACCAACCTGTCCAA } \\
\text { GTTC }\end{array}$ \\
\hline \multirow[t]{2}{*}{ MLC2V } & Forward & GACCCAGATCCAGGAGTTCA \\
\hline & Reverse & AATTGGACCTGGAGCCTCTT \\
\hline \multirow[t]{2}{*}{ NCX1 } & Forward & TGTGCATCTCAGCAATGTCA \\
\hline & Reverse & TGATGCCAATGCTCTCACTC \\
\hline \multirow[t]{2}{*}{ SERCA2A } & Forward & ACCCACATTCGAGTTGGAAG \\
\hline & Reverse & CAGTGGGTTGTCATGAGTGG \\
\hline \multirow[t]{2}{*}{ RYR2 } & Forward & CGTTCTAACCAGCATCTCATC \\
\hline & Reverse & CGAGCAATACAACCTGACC \\
\hline \multirow[t]{2}{*}{ CaSQ } & Forward & GAGCTTGTGGCCCAGGTCCT \\
\hline & Reverse & GATCTCCACTGGGTCTTCAA \\
\hline \multirow[t]{2}{*}{ Triadin } & Forward & TCAGTTGCTCCACACTGAGC \\
\hline & Reverse & CCCATTTACAGACGGGAAAC \\
\hline \multirow[t]{2}{*}{ Junctin } & Forward & GTAAAATGGCATCCCGAGAC \\
\hline & Reverse & GGATGATGATGCCAGAGC \\
\hline \multirow[t]{2}{*}{ S16 } & Forward & AAGCCCTGGTGGCCTATTAC \\
\hline & Reverse & CCAAACTTTTTGGACTCGCAGCG \\
\hline
\end{tabular}

Expression of the genes of interest was evaluated by endstage or real-time quantitative amplification using $0.2 \mu \mathrm{L}$ of the cDNA as a template using the respective oligonucleotides (see Tables 1 and 2). Quantitative PCR analysis was performed with DNA engine opticon 2 real-time PCR Detection System (Bio-Rad, Hercules, CA) using the iQ SYBR Green Supermix (Bio-Rad). For amplification, after initial holds for $5 \mathrm{~min}$ at $50^{\circ} \mathrm{C}, 50$ cycles of $95^{\circ} \mathrm{C}$ for $15 \mathrm{sec}$, followed by $57^{\circ} \mathrm{C}$ for $30 \mathrm{sec}$ and $72^{\circ} \mathrm{C}$ for $30 \mathrm{sec}$, melt curve analysis was performed. The relative quantification of the PCR products was performed according to the $2^{-\Delta \Delta \mathrm{Ct}}$ method, using ribosomal protein S16 (S16) as internal control. Where $\Delta \Delta \mathrm{Ct}=\left[\left(\mathrm{Ct}_{\text {target gene }}-\mathrm{Ct}_{\mathrm{S} 16}\right)_{\mathrm{CoCl}_{2} \text { treated group }}-\left(\mathrm{Ct}_{\text {target gene }}-\right.\right.$ $\left.\left.\mathrm{Ct}_{\mathrm{S16}}\right)_{\text {control group] }}\right]$.

\section{Flow cytometry analysis}

The percentage of ESC-derived cardiomyoyctes was quantified by FACS analysis. Briefly, EBs were dissociated with $1 \mathrm{mg} / \mathrm{mL}$ collagenase B (Roche Applied Sciences, Penzberg, German), washed with differentiated medium, and the cells permeabilized by Cytofix/Cytoperm permeabilization kit (BD Biosciences, San Diego, CA). Cells were then stained with monoclonal antisarcomeric actinin antibody (A7811, Sigma, St. Louis, MO) using antimouse IgG $\mathrm{H}+\mathrm{L}-\mathrm{PE}$ as a secondary antibody stain (Beckman Coulter, Fullerton, CA). Analysis was performed with a Beckman Coulter FC500 flow cytometer in which at least 1000 events were counted.

\section{Isolation of hES2-derived cardiomyocytes}

Isolation of hES2-derived cardiomyocytes has been described previously (Au et al., 2009; Lieu et al., 2009; Liu et al., 2007, 2009). Briefly, beating outgrow ths were microsurgically dissected from hES2-derived EBs (25 days) with a glass knife, followed by incubation in collagenase B $(1 \mathrm{mg} / \mathrm{mL}$, Roche Applied Sciences) with DNase I (60 U/mL, Roche Applied Sciences) at $37^{\circ} \mathrm{C}$ for $30 \mathrm{~min}$ with occasional rocking. Isolated cells were recovered in Kraftbrühe (KB) solution containing $(\mathrm{mM}) 85 \mathrm{KCl}, 30 \mathrm{~K}_{2} \mathrm{HPO}_{4}, 5$ $\mathrm{MgSO}_{4}, 1$ EGTA, $2 \mathrm{Na}_{2}$-ATP, 5 pyruvic acid, 5 creatine, 20 taurine, and $20 \mathrm{D}$-glucose at room temperature for $1 \mathrm{~h}$. The cells were subsequently plated on $0.1 \%$ gelatin coated glass cover slips in 24-well culture plates with corresponding differentiation medium. Immunohistochemistry, electrophysiological study, and confocal calcium imaging of isolated cells or cell clusters were performed within 2 days.

\section{Immunohistochemistry}

The hES2-derived cardiomyocytes were fixed with $2 \%$ paraformaldehyde for $15 \mathrm{~min}$ at $4^{\circ} \mathrm{C}$ followed by two washes with phosphate-buffered saline (PBS). Prior to overnight incubation with murine monoclonal anticardiac Troponin-T antibody (NeoMarker, Fremont, CA) or monoclonal antisarcomeric actinin antibody (A7811, Sigma) at $4^{\circ} \mathrm{C}$, fixed cells were blocked in $2 \%$ bovine serum alubmin (BSA), PBS, and $0.1 \%$ Triton X-100 for $2 \mathrm{~h}$. After removal of unbound antibodies with washing buffer, cells were incubated with Alexa-488 conjugated antimouse secondary antibodies (Invitrogen, Carlsbad, CA) (1:100 dilution in washing buffer) for $1 \mathrm{~h}$ at room temperature. Cells were then mounted on glass slides with a mounting medium containing DAPI. The presence of the immune complex was examined by fluorescent microscopy.

\section{Electrophysiological study}

For electrophysiological characterization of hES2-derived cardiomyocytes, the standard whole-cell patch-clamp recording was performed at $37 \pm 0.5^{\circ} \mathrm{C}$ to record the action potential phenotypes (HEKA Instruments Inc., Southboro, MA) as previously described (Chan et al., 2009). In brief, patch pipettes were prepared from $1.5-\mathrm{mm}$ thin-walled borosilicate glass tubes using a Sutter micropipette puller P-97 and had typical resistances of 3-4 M $\Omega$ when filled with an 
internal solution containing $(\mathrm{mM}): 110 \mathrm{~K}^{+}$aspartate, $20 \mathrm{KCl}$, $1 \mathrm{MgCl}_{2}, 0.1 \mathrm{Na}-\mathrm{GTP}, 5 \mathrm{Mg}$-ATP, $5 \mathrm{Na}_{2}$-phosphocreatine, 5 EGTA, 10 HEPES, and $\mathrm{pH}$ adjusted to 7.3 with $\mathrm{KOH}$. The external Tyrode's bath solution consisted of (mM): $140 \mathrm{NaCl}$, $5 \mathrm{KCl}, 1 \mathrm{MgCl}_{2}, 0.4 \mathrm{KH}_{2} \mathrm{PO}_{4}, 1.8 \mathrm{CaCl}_{2}, 10$ Glucose, 5 HEPES, with $\mathrm{pH}$ adjusted to 7.4 with $\mathrm{NaOH}$. For those hES2-derived cardiomyocytes that were electrically quiescent, a stimulation of $0.1-1 \mathrm{nA}$ for $5 \mathrm{msec}$ was given to elicit an action potential. The sampling frequency was $2.00 \mathrm{kHz}$, and data were corrected for the liquid junction potentials of $+20.3 \mathrm{mV}$.

\section{Confocal calcium imaging}

Confocal calcium imaging was performed with the protocol described previously (Ng et al., 2010, 2011). In brief, the isolated hES2-derived cardiomyocytes were loaded with $5 \mu \mathrm{M}$ Fluo-3 AM (Invitrogen) for $25 \mathrm{~min}$ at $37^{\circ} \mathrm{C}$ in Tyrode solution containing $140 \mathrm{mM} \mathrm{NaCl}, 5 \mathrm{mM} \mathrm{KCl}, 1 \mathrm{mM} \mathrm{MgCl}$, $1.8 \mathrm{mM} \mathrm{CaCl}_{2}, 10 \mathrm{mM}$ glucose, and $10 \mathrm{mM}$ HEPES at $\mathrm{pH}$ 7.4. Spontaneous calcium transient of single or clusters of ESCderived cardiomyocytes was recorded with a confocal imaging system (Olympus Fluoview System version 4.2 FV300 TIEMPO) mounted on an upright Olympus microscope (IX71). To record the caffeine-induced calcium transient, $20 \mu \mathrm{L}$ of $10 \mathrm{mM}$ caffeine was applied onto the cell surface during measurement. To evaluate the effect of ryanodine, spontaneous calcium transients were recorded before and after the incubation ( $5 \mathrm{~min})$ of Tyrode solution containing $10 \mu \mathrm{M}$ ryanodine. The calcium transient was quantified as the background subtracted fluorescence intensity changes normalized to the background subtracted baseline fluorescence. Data were fed into the Felix 32 (PTI) software for analysis.

\section{Statistical analysis}

Continuous variables are expressed as mean \pm SEM. Statistical comparisons were performed using a Student's $t$-test. Calculations were performed with GraphPad Prism 5
(GraphPad, LaJolla, CA). A p-value $<0.05$ was considered statistically significant.

\section{Results}

\section{Cobalt chloride treatment increases the protein levels} of HIF-1 $\alpha$ in hES2 human ESCS

To evaluate the possibility of using cobalt chloride to activate the HIF-1 pathway in hES2 cells, expression of the HIF-1 pathway components in undifferentiated hES2 cells was evaluated using end-stage PCR analysis. As shown in Figure 1A, both HIF$1 \alpha$ and HIF- $1 \beta$ subunits as well as HIF- $2 \alpha$ were expressed after cobalt chloride treatment but there was no detectable HIF- $3 \alpha$. These findings suggest that cobalt chloride treatment activates expression of the HIF- $1 \alpha$ and HIF- $2 \alpha$ signal in hES2 cells. The expression of other components of the HIF-1 pathway, including the prolyl hydroxylases termed oxoglutarate-dependent proline 4-hydroxylases, EGLN-1 to 3 were also observed.

Undifferentiated hES2 cells were then subjected to treatment with cobalt chloride $(50 \mu \mathrm{M})$ or reduced oxygen $(5 \%$ $\mathrm{O}_{2}$ ) followed by Western blot analysis to evaluate the protein levels of HIF- $1 \alpha$. As shown in Figure 1B, both $72 \mathrm{~h}$ of cobalt chloride treatment and $24 \mathrm{~h}$ of reduced oxygen treatment remarkably increased cell HIF- $1 \alpha$ protein levels. Nonetheless, the cell viability of the reduced oxygen-treated groups was thus significantly reduced to about $50 \%$ (Fig. 1C). There was no such reduction in cell viability in the control $\left(21 \% \mathrm{O}_{2}\right)$ or cobalt chloride-treated group (Fig. 1C) and no significant differences in the mRNA levels of HIF-1 $\alpha$ between the two groups (Fig. 1D). This suggests that cobalt chloride prevents the breakdown of HIF- $1 \alpha$ protein rather than increasing the gene expression of HIF- $1 \alpha$ in the undifferentiated hES2 cells.

\section{Cobalt chloride treatment initiates the differentiation of undifferentiated hES2 cells}

To evaluate the effects of cobalt chloride-induced HIF-1 $\alpha$ stabilization on initiating cardiac differentiation, cultured
FIG. 1. Effect of cobalt chloride on undifferentiated hES2 ESCs. (A) The expressions of the major components of the hypoxia inducible factor 1-pathaway in untreated undifferentiated hES2 stem cells as revealed by end-stage PCR analysis. (B) Undifferentiated hES2 cells were treated with $50 \mu \mathrm{M}$ cobalt chloride $\left(\mathrm{CoCl}_{2}\right)$ for $72 \mathrm{~h}$ or cultured in $5 \%$ oxygen for $24 \mathrm{~h}$. After treatment, the protein levels of HIF- $1 \alpha$ were determined by Western blot analysis with HIF- $1 \alpha$ specific antibody, while $\beta$ actin protein levels were used as internal control. (C) The viabilities of cells after cobalt chloride or reduced oxygen treatments were evaluated with Trypan Blue dye exclusion assay. (D) The relative mRNA levels of HIF- $1 \alpha$ as revealed by real-time quantitative PCR using S16 as internal reference. Results are presented in mean \pm SEM from at least three independent experiments, where ${ }^{* *} p<0.005$ compared to the control group, and \#\#p<0.005 compared to the cobalt chloride-treated group.

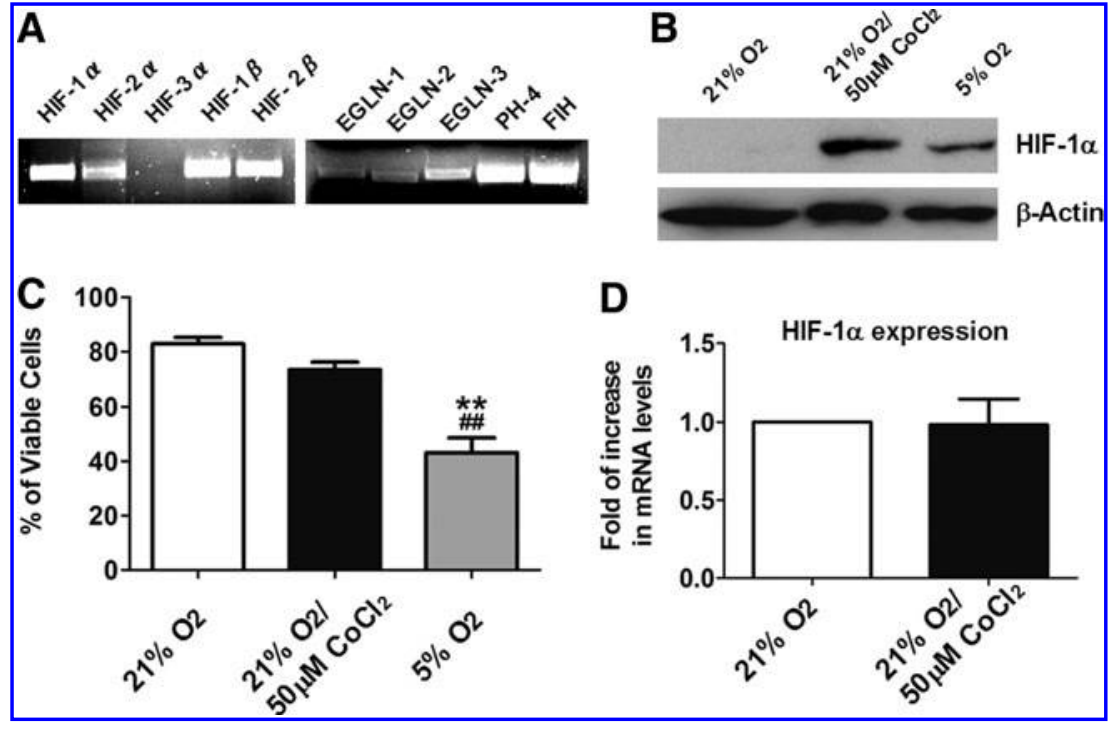


hES2 cells were harvested immediately following cobalt chloride treatment, and the expression of early cardiacspecific transcription factors [GATA-binding protein 4 (GATA4) and NK2 transcription factor related locus 5 (Nkx2.5)] and cardiotrophic factors [vascular endothelial growth factor (VEGF) and cardiotrophin-1 (CT1)] were compared with controls using quantitative real-time PCR analysis. Cobalt chloride pretreatment significantly increased mRNA levels of Nkx2.5 (1.8-fold, $p<0.05)$, VEGF (1.6-fold, $p<.05$ ), and CT1 (4.3-fold, $p<0.005$ ), but not GATA4 (Fig. 2A). Interestingly, in addition to the early cardiac markers, the expression of the early neuronal marker, neurogenin1 (NGN) was also increased significantly $(3.1$-fold, $p<0.005)$ upon cobalt chloride treatment, whereas those governing the development of skeletal muscle (MYOG) and erythropoietin lineage (GATA1 and SPI1) did not show significant changes (Fig. 2A).

On the other hand, the expressions of the pluripotency markers, octamer-binding transcription factor 4 (OCT4), sex determining region Y-box 2 (SOX2), and homeobox protein NANOG (NANOG) were significantly reduced by 0.76 -fold $(p<0.005), 0.65(p<0.05)$, and $0.38(p<0.005)$, respectively, after cobalt chloride treatment (Fig. 2B).

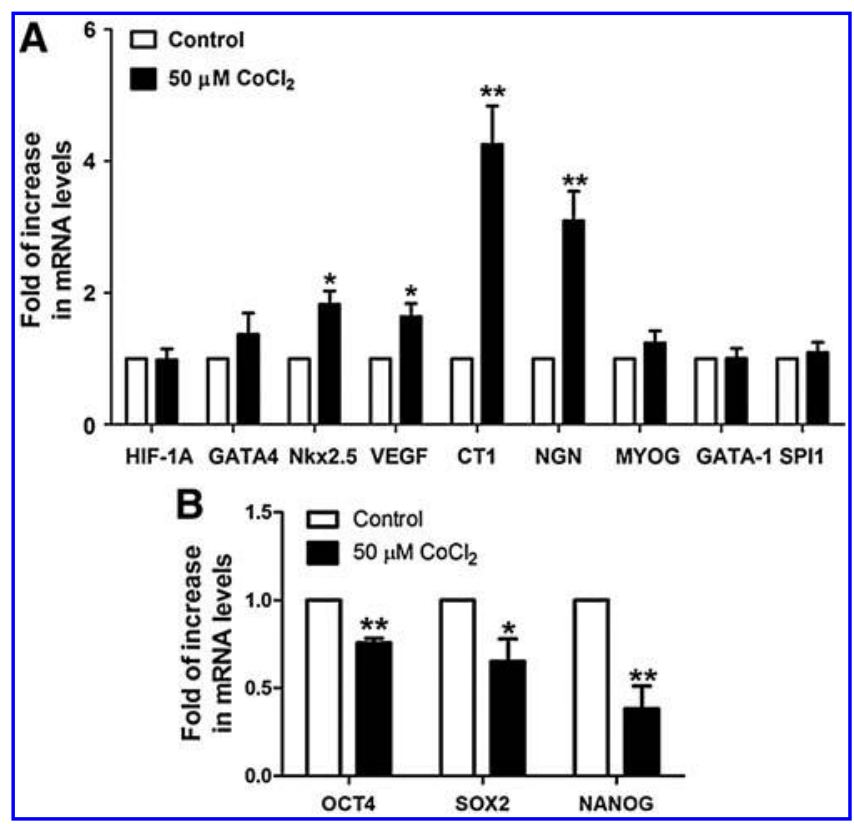

FIG. 2. Cobalt chloride treatment increased the mRNA levels of cardiogenic transcription factors, cardiotrophic factors, and neuronal transcription factor. (A) To evaluate the action of cobalt chloride in initiating cardiac differentiation of human ESCs, undifferentiated hES2 cell were cultured in the presence or absence of $50 \mu \mathrm{M}$ cobalt chloride $\left(\mathrm{CoCl}_{2}\right)$, and the expressions of GATA4, Nkx2.5, VEG, CT1, NGN, MYOG, GATA1, and SPI1 were compared by quantitative real-time PCR analysis using the ribosomal protein S16 as internal reference. (B) The relative mRNA levels of OCT4, SOX2, and NANOG as revealed by real-time quantitative PCR using S16 as an internal reference. Results are presented in mean $\pm S E M$ from at least three independent experiments, where ${ }^{*} p<0.05$ and ${ }^{* *} p<0.005$ compared to the control groups.

\section{Cobalt chloride pretreatment facilitate the cardiac differentiation of hES2 human ECSs}

The control and cobalt chloride-pretreated cells were then allowed to undergo cardiac differentiation. Comparison at the 20th day after plating revealed that the percentage of beating embryoid bodies (EBs) (Fig. 3A; $p<0.05$ ) and sarcomeric-actinin-positive cells (Fig. 3B; $p<0.005$ ) was significantly higher following cobalt chloride treatment. The mRNA level of the cardiac specific genes, that is, myosin heavy chain (MHC)-A (1.7-fold, $p<0.05)$; MHC-B (1.8-fold, $p<0.05)$, and myosin light chain 2V (MLC2V) (1.5-fold, $p<0.05)$ in the EBs differentiated from the cobalt-treated hES2 cells was also significantly higher (Fig. 3C). Immunohistochemistry demonstrated that the cardiomyocytes (cardiac troponin-T positive and sarcomeric-actinin-positive cells) differentiated from the cobalt chloride-treated group were generally more elongated (a significantly higher length/width ratio) than those from the control group (Fig. $4 \mathrm{~A}$ and $\mathrm{B})$. At higher magnification, a more organized pattern of myofibrils could be observed in the cobalt chloridepretreated group. These results suggest that cobalt chloride pretreatment facilitates cardiac differentiation of the undifferentiated hES2 cells.

\section{Electrophysiology of hESC-derived cardiomyocytes}

To test whether cobalt-induced cardiac differentiation influences the maturation of hES2-derived cardiomyocytes, their electrophysiological properties were characterized by whole-cell patch clamp experiments. Figure 5 depicts the electrophysiogical characteristics (i.e., ventricular-like, atriallike, and nodal-like) and the corresponding representative action potential recordings of cardiomyocytes derived from the control and cobalt chloride-treated hES2. In the control group, out of seven cells analyzed, $71 \%$ showed ventricularlike phenotype and 29\% exhibited the atrial-like phenotype (Fig. 5A, left panel). Despite the limited number of cells being studied, this observation is inline with the previous study (Moore et al., 2008). In the cobalt chloride-pretreated group, of 24 cardiomyocytes measured, $67 \%$, 25\%, and $8 \%$, respectively demonstrated atrial-, ventricular-, and nodal-like phenotype (Fig. 5A, right panel). When the action potentials at $90 \%$ (APD90s) and 50\% (APD50s) repolarization of both groups were compared, ventricular-like cells in particular showed a decrease in both APD90s $(p<0.05)$ and APD50s $(p<0.01)$ on cardiomyocytes of the cobalt chloride-pretreated group (Fig. 5B and C). In addition, maximum rise of the action potential upstrokes $\left(d V / d t_{\max }\right)$ for cardiomyocytes showing atrial- and ventricular-like phenotypes were both significantly increased $(p<0.05)$ (Fig. 5D). On the other hand, the maximum diastolic potentials (MDPs) of both atrial-like and ventricular-like cells showed no significant difference between the two groups (Fig. 5E).

\section{Cardiomyocytes differentiated from cobalt chloride-pretreated cells exhibit improved calcium-handling ability}

Spontaneous intracellular calcium $\left(\mathrm{Ca}^{2+}\right)$ transients in the hES2-derived cardiomyocytes were assessed using confocal laser microscopy 25 to 30 days following onset of beating. In both control and cobalt chloride-pretreated groups, $\mathrm{Ca}^{2+}$ 
FIG. 3. Effect of cobalt chloride pretreatment on the cardiac differentiation of hES2 human ESCs. (A) The undifferentiated hES2 cells were cultured for $72 \mathrm{~h}$ in the presence or absence of 50 [M cobalt chloride $\left.\left(\mathrm{CoCl}_{2}\right)\right]$ followed by cardiac differentiation. The resulting embryoid bodies (EBs) were allowed to attach onto gelatin-coated plates and the occurrence of beating EBs were observed and counted for 20 to 25 days starting from the day of attachment. (B) After the 25-day period of cardiac differentiation, the EBs were collected for RNA isolation and the expressions of cardiac specific markers, MHC-A, MHC-B, and MLC2V between the control and cobalt chloride-pretreated groups were compared by real-time quantitative PCR analysis. (C) Flow cytometry analysis of sarcomeric actinin-positive cells. Results are presented in mean \pm SEM from at least three independent experiments, where ${ }^{*} p<0.05$ and ${ }^{* *} p<0.005$ compared to the control groups.
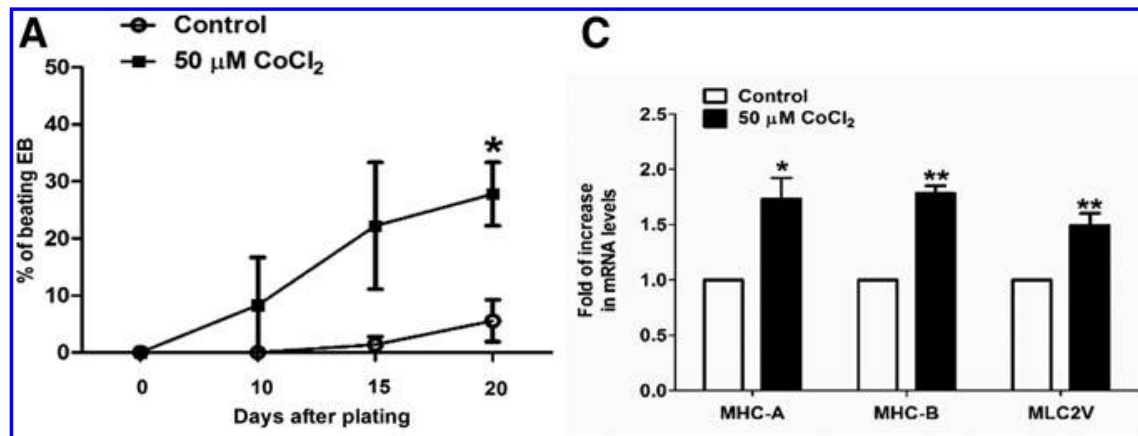

B
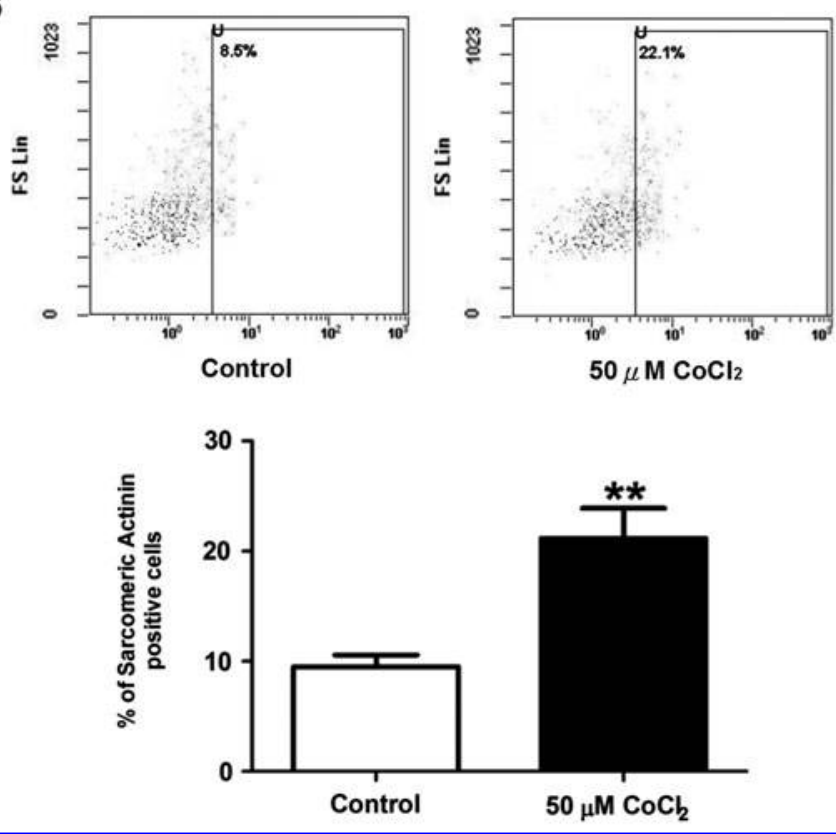

flux was observed in their derived cardiomyocytes. Fluorescence intensity values measured against time revealed that the amplitude of $\mathrm{Ca}^{2+}$ transient measured in cardiomyocytes derived from the cobalt chloride-pretreated hES2 cells was approximately 2.5-fold higher than that in the untreated hES2-derived cardiomyocytes (Fig. 6A and B; $p<0.05$ ). Similarly, kinetic analysis revealed that cardiomyocytes derived from cobalt chloride-pretreated hES2 cells displayed faster calcium release and reuptake rates as indicated by the significant increase in the upstroke ( $\left.V_{\text {maxupstroke }}\right)$ and decay $\left(V_{\text {maxdecay }}\right)$ velocities (Fig. 6C and $\mathrm{D} ; p<0.05$ ).

To evaluate the sarcoplasmic reticulum performance, the caffeine-induced $\mathrm{Ca}^{2+}$ transient was also recorded. As shown in Figure 6E, brief exposure to $10 \mathrm{mM}$ caffeine induced a surge in cytosolic $\mathrm{Ca}^{2+}$ that subsequently decayed back to baseline in the cardiomyocytes derived from both control and cobalt chloride-pretreated hES2 cells. Similar to the observations in spontaneous $\mathrm{Ca}^{2+}$ transient, the amplitude of the caffeine-induced $\mathrm{Ca}^{2+}$ from the cobalt chloridepretreated group was significantly higher compared with the control group (Fig. 6F, approximately 2.4-fold increase; $p<0.01)$. This finding suggests that sarcoplasmic reticulum $\mathrm{Ca}^{2+}$ storage is increased in cardiomyocytes derived from cobalt chloride-pretreated hES2 cells. In the kinetic analysis, cardiomyocytes derived from cobalt chloride-pretreated
hES2 cells also displayed faster upstroke and decay velocities during caffeine-induced $\mathrm{Ca}^{2+}$ transient (Fig. 6G and H). In addition, application of $10 \mu \mathrm{M}$ ryanodine significantly reduced the amount and release rate of intracellular calcium in these cardiomyocytes, but not those from the control group (Fig. 6I and J).

In cardiomyocytes, the sodium calcium exchanger (NCX1), cardiac sarcoplasmic/endoplasmic reticulum calcium ATPase (SERCA2A), and cardiac specific ryanodine receptor (RYR2) are the key components that mediate calcium transport (Graham et al., 2004). Calsequestrin (CaSQ), triadin, and junctin play a role in regulating the sarcoplasmic reticulum function via their binding to RYR2 (Bers, 2004; Fan et al., 2008; Knollmann, 2009). Real-time quantitative PCR analysis revealed that the expression of SERCA2A (3.6-fold, $p<0.05)$, RYR2 (4.1-fold, $p<0.05)$, and triadin (2.5-fold, $p<0.05)$ was significantly increased in EBs derived from the cobalt chloride-pretreated hES2 cells compared with control cells (Fig. 7). Nevertheless, there was no significant change in the mRNA level of NCX1, CaSQ, or junctin in the two groups. These findings suggest that improved sarcoplasmic reticulum function of cardiomyocytes derived from cobalt chloride-pretreated hES2 cells is likely related to increased expression of the $\mathrm{Ca}^{2+}$ handling components. 

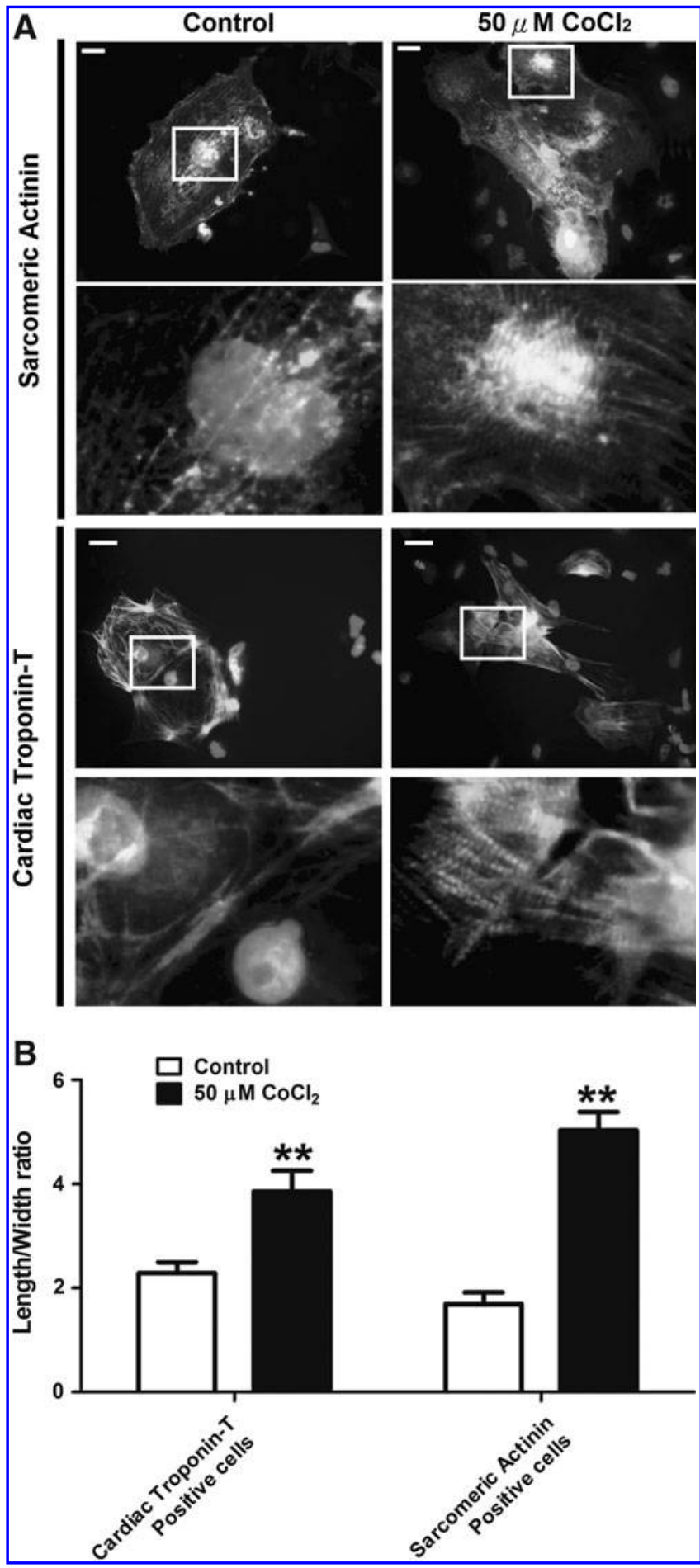

FIG. 4. Immunohistochemistry of hES2-derived cardiomyocytes. (A) Individual cardiomyocytes was isolated from the beating area of the EBs were paraformaldehyde fixed and stained with the cardiomyocytes specific markers cardiac troponin-T and sarcomeric actinin. Lower panel represent the enlarged view of the area of interest (squared region). Scale bar $=25 \mu \mathrm{mM}$. (B) the length to width ratio of cardiac troponin-T and sarcomeric actinin positive cells. Results are presented as mean \pm SEM from the measurement of 25 to 30 cells from each groups, where ${ }^{* *} p<0.005$ compared to the control groups.

\section{Discussion}

Our recent study has demonstrated that the cardiac differentiation and maturation of murine ESCs can be promoted by exogenous expression of the full-length HIF- $1 \alpha$ cDNA under a constitutive EF1 $\alpha$ promoter ( $\mathrm{Ng}$ et al., 2010). These observations highlight the potential importance of the HIF- $1 \alpha$ subunit in directing cultured ESCs to differentiate into more mature cardiomyocytes. Nevertheless, the therapeutic application of this strategy is limited by the requirement of a viral vector. The present study aimed to evaluate the use of cobalt chloride-induced HIF- $1 \alpha$ stabilization to promote cardiac differentiation of cultured human ESCs. The use of divalent ions (such as cobalt, iron, or nickel compounds) has a potential advantage over the reduced oxygen tensions required in stabilizing the HIF- $1 \alpha$ subunit. First, the substantial cost involved in the setup and operation of a reduced oxygen culture system is avoided. Second, the use of a pharmacological treatment that includes the defined concentration of drugs or chemicals is also anticipated to provide more robust and reproducible results between different laboratories. This is especially important given that the actual partial pressure of oxygen that cells experience may differ widely among different culture setups and sources of gas. As shown in this study, treatment of undifferentiated hES2 human ESCs with cobalt chloride for 3 days was associated with remarkably increased protein levels of HIF- $1 \alpha$. Although increased HIF- $1 \alpha$ expression was also observed in cells cultured under reduced oxygen conditions, a decrease in cell viability was also evident in this study. In contrast, previous studies showed that reduced low oxygen tension can enhance the culture and maintenance of H1 human ESCs in undifferentiated states (Ezashi et al., 2005). The reasons for this discrepancy remain unclear, but may be related to different ESC lines and culture systems. In this study, the HES2 human ESC line was cultured in a feeder-free serum-free culture system. Previous studies (Ezashi et al., 2005) have used a mouse embryonic fibroblast as feeder layer supplemented with $20 \%$ KnockOut serum replacer to culture H1 human ESC line.

In this study, cobalt chloride pretreatment notably increased the occurrence of beating EBs as well as expression of cardiac specific structure components (MHC-A, MHC-B, and MLC2V) over the 25-day period of cardiac differentiation compared with controls. As cardiac differentiation of ESCs was performed in the absence of cobalt chloride, it is reasonable to speculate that cobalt chloride help initiate the early cardiac differentiation process before embryoid body formation, and this could be evidenced by the downregulation of the pluripotent markers (OCT4, SOX2, and NANOG), and the upregulation of early cardiac differentiation factors Nkx2.5, VEGF, and notably CT1 gene expression during the pretreatment period. It is interesting that the expression of NGN, an early neuron-specific marker, was also significantly increased after cobalt chloride treatment, while the expressions of the skeletal muscle specific (MYOG) and hematopoietic markers (GATA1 and SPI1) shows no significant difference. The reasons account for such observation is not clear but deserve further investigation that would be out of the scope of the present study.

HIF- $1 \alpha$ plays an important role in the embryonic heart development (Iyer et al., 1998; Nagao et al., 2008; RamirezBergeron and Simon, 2001), yet the direct action of HIF- $1 \alpha$ on the promoters of many cardiac genes remains to be 


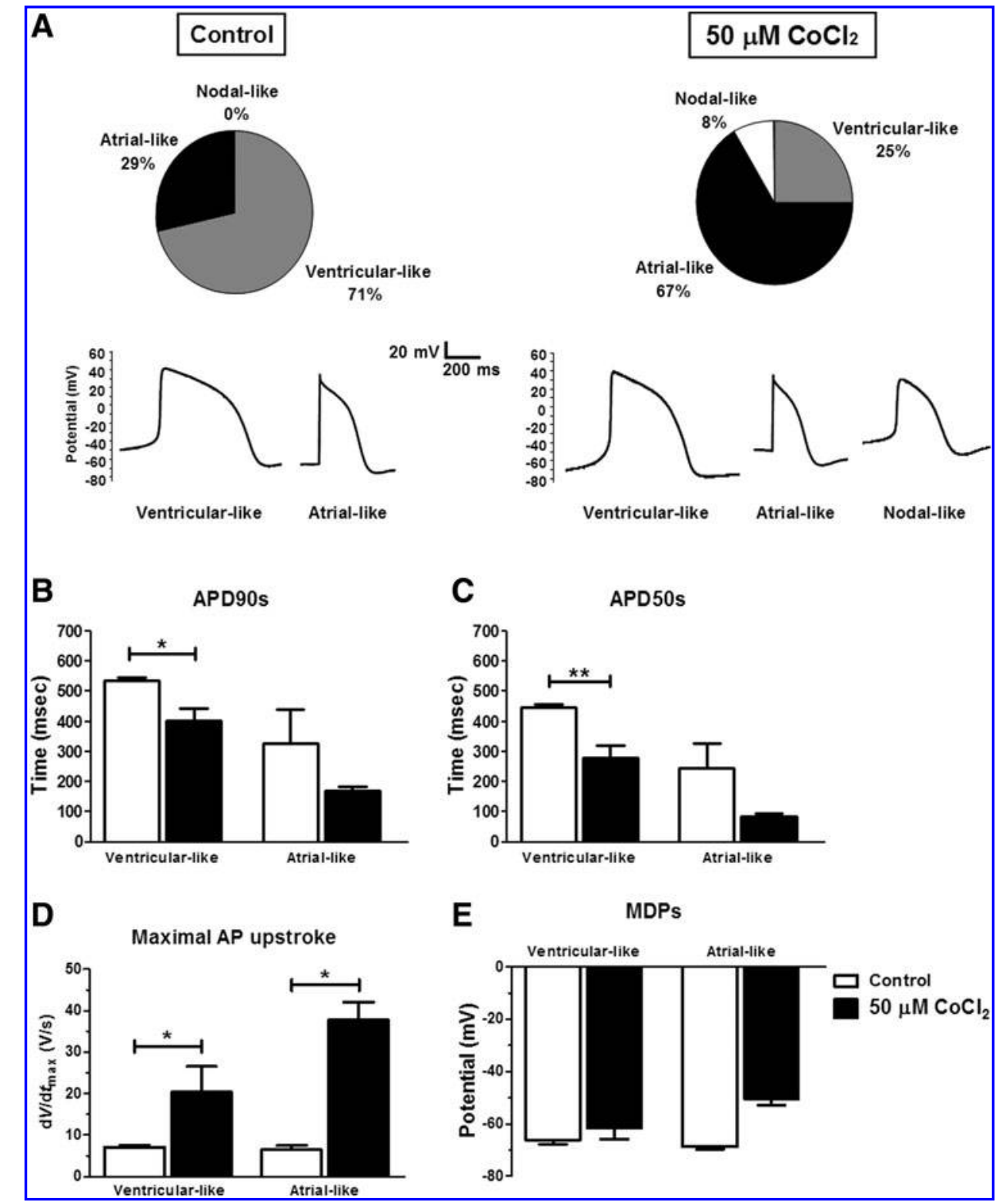

FIG. 5. (A) Cell-type distribution and the corresponding representative action potential tracings of the cardiomyocytes derived from control (left panel) and cobalt-pretreated (right panel) hES2 cells. Action potentials at $90 \%$ (APD90s) and 50\% (APD50s) repolarization (B, C), maximal action potential (AP) upstroke (D) and the maximal diastolic potentials (MPDs) (E) of hES2-derived cardiomyocytes from control (six cells) and cobalt chloride-pretreated groups (24 cells). Results are presented as mean \pm SEM $\left({ }^{*} p<0.05 ;{ }^{* *} p<0.005\right)$. determined. Recently, Nagao et al. (2008) described that the knockdown of HIF- $1 \alpha$ causes significant reduction in the nkx2.5 expression in a Xenopus model (Nagao et al., 2008) and this may explain, in part, our observations that cobalt chloride increase NKX2.5 expression in the undifferentiated hES2 cells.

The importance of VEGF and CT1 in the mediation of cardiac differentiation from pluripotent stem cells has been highlighted in various studies (Ateghang et al., 2006; Chen et al., 2006; Xinyun et al., 2010). The appearance of a population of kinase insert domain protein receptor (KDR)positive progenitor cells during the very initial period of differentiation dictates the subsequent cardiac differentiation events of the ESCs (Yang et al., 2008). Accordingly, increased expression of VEGF during cobalt chloride pretreatment may play a role in activation of the KDR-positive cardiac progenitor cells to increase the occurrence of beating EBs and the percentage of sarcoplasmic actinin-positive cardiomyocytes. It should be noted that attempts have been made to supplement cobalt chloride throughout the whole differentiation period, but such an approach appears to reduce the number of beating EBs (data not shown). The underlying reason is unclear but may be related to the oxidative toxicity that arises from prolonged cobalt chloride treatment.

When the cobalt chloride-pretreated groups was compared with the control group, the former showed a notable increase not only in the differentiation of atrial- and nodallike cardiomyocytes, but also the maximum rise of the action potential upstroke $\left(d V / d t_{\max }\right)$. Such a significant increase in the $d V / d t_{\max }$ reflects an improved electrical maturity after cobalt chloride treatment. Nonetheless, the limited sample size means further work is required to identify whether cobalt chloride pretreatment determines the fate of cadiomyogenesis. In addition to these electrophysiological observations, calcium homeostasis of the hES2-derived cardiomyocytes was also studied. Those derived from the cobalt chloride-pretreated group exhibited significant increases in the amplitude, upstroke rate, and decay rates of the calcium transient compared with the control group. This indicates 
that the former may more effectively handle the release and reuptake of intracellular calcium during excitationcontraction coupling. In addition, the increased performance of caffeine-induced calcium transient and increased ryanodine sensitivity in the cobalt chloride-pretreated cells suggests these cardiomyocytes have improved sarcoplasmic reticulum
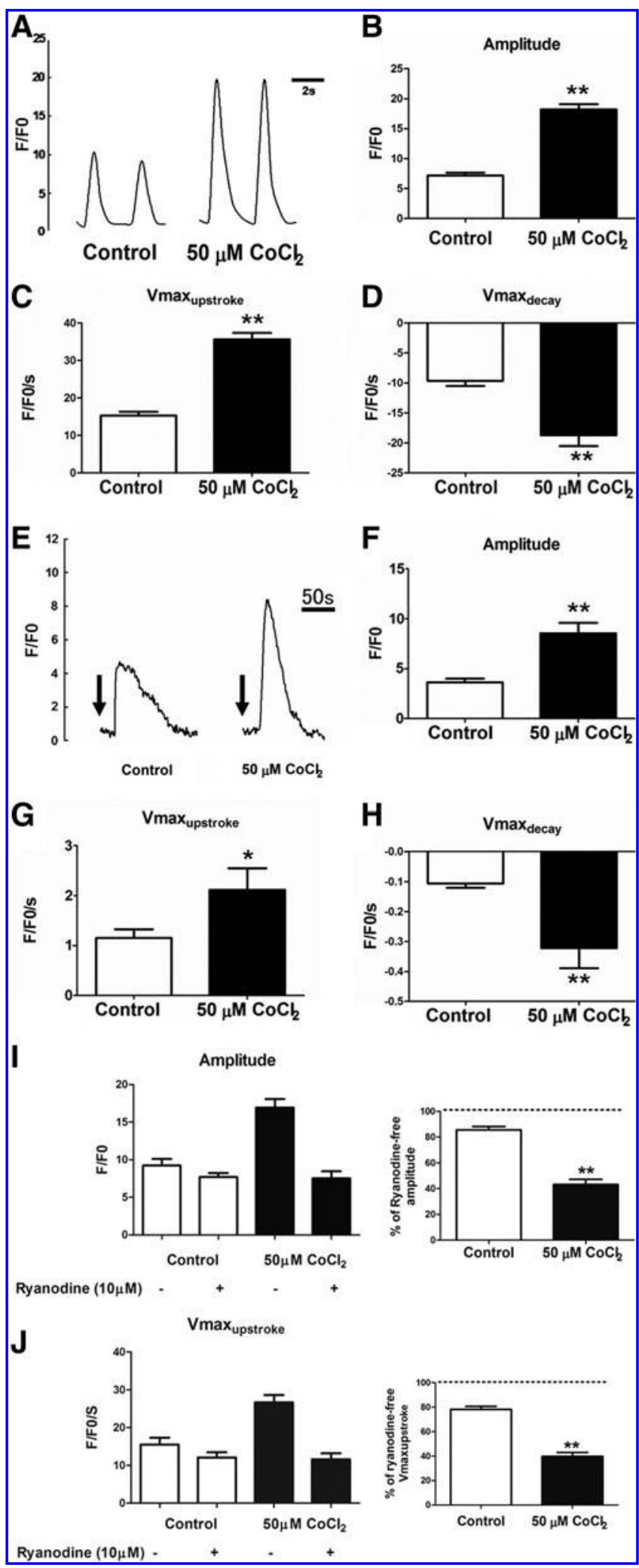

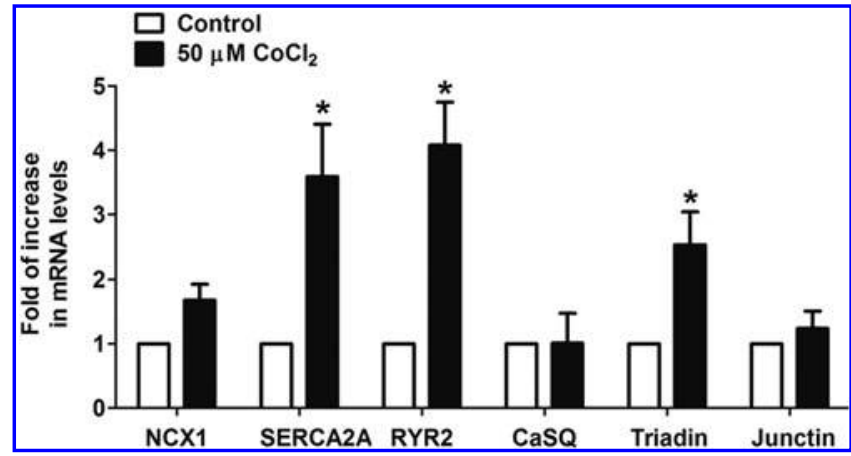

FIG. 7. The expression of calcium-handling components in cardiomyocytes. The mRNA levels of calcium-handling components NCX1, SERCA2A, RYR2, CaSQ, Triadin, and Junctin in the EBs derived from the control or hES2 cells pretreated with $50 \mu \mathrm{M}$ cobalt chloride $\left(\mathrm{CoCl}_{2}\right)$ were evaluated by real-time quantitative PCR analysis using GATA4 as an internal reference. Data shown as mean \pm SEM from at least three independent experiments; ${ }^{*} P<0.05$.

function. This assumption was further supported by the observation that both SERCA2A and RYR2, the two key components that mediate the sequestration and release of calcium from the sarcoplasmic reticulum, respectively, were significantly upregulated in the cobalt chloride-pretreated cells. In addition to SERCA2A and RYR2, the expression of RYR2 regulatory components dictates the maturity of the ESC-derived cardiomyocytes (Liu et al., 2007, 2009). Although there was no observable change in the expression of CaSQ and junctin between the two groups, a significant increase in the mRNA levels of triadin was also found in the cobalt chloridepretreated group: this might contribute to the improved sarcoplasmic reticulum-mediated calcium transient in the treated cells. With increasing evidence of the importance of sarcoplasmic reticulum calcium load and transient in contractile function of ESC-derived cardiomyocytes (Fu et al., 2006;

FIG. 6. (A) Representative tracings of rhythmic spontaneous $\mathrm{Ca}^{2+}$ transients showing in cardiomyocytes derived from the control and cobalt-pretreated hES2 cells. (B) Amplitude. (C) Maximal upstroke velocity ( $V_{\max }$ upstroke). (D) Maximal decay velocity ( $V_{\max }$ decay) of $\mathrm{Ca}^{2+}$ transients in the hES2-derived cardiomyocytes. (E) Representative tracings of caffeine-induced $\mathrm{Ca}^{2+}$ release from sarcoplasmic reticulum in the cardiomyocytes derived from the control and cobalt-pretreated hES2 cells, demonstrating caffeine-sensitive $\mathrm{Ca}^{2+}$ stores and fractional release of total sarcoplasmic reticulum $\mathrm{Ca}^{2+}$ load. (F) Amplitude. (G) Maximal upstroke velocity ( $V_{\max }$ upstroke). (H) Maximal decay velocity ( $V_{\max }$ decay) of $\mathrm{Ca}^{2+}$ transients in the hES2-derived cardiomyocytes. Effects of ryanodine on the calcium release $(\mathbf{I}, \mathbf{J})$, the amplitude (I), and maximal upstroke velocity (J) of spontaneous calcium transients from individual cardiomyocytes were recorded in the absence or presence of $10 \mu \mathrm{M}$ ryanodine. The percentage of amplitudes and $V_{\max }$ upstroke values under ryanodine application to the corresponding values recorded under ryanodine-free conditions were showed in the right panels. Data shown as mean \pm SEM from the recordings of 20 to 25 cells / cell clusters from three to five independent experiments $\left({ }^{*} p<0.05 ;{ }^{* *} p<0.005\right)$. 
Liu et al., 2007), it has become clear that the proper calciumhandling property of the ESC-derived cardiomyocytes is crucial to functional integration of the transplanted cells into the host tissue (Liu et al., 2007).

In conclusion, our present study provides evidence that cobalt chloride-induced HIF- $1 \alpha$ stabilization facilitates the cardiac differentiation of human ESCs in a serum- and feeder-free system. Unlike low oxygen culture systems, no sophisticated or costly facilities are required, and there is no reduction in viability of cobalt chloride-treated human ESCs. Cardiomyocytes derived from cobalt chloride-treated cells also appear to have a more advanced calcium-handling ability. This may be due to increased expression of the sarcoplasmic reticulum calcium-handling components. These data provide a novel strategy to encourage maturation of calcium handling of cardiomyocytes from ESCs for therapeutic application.

\section{Acknowledgments}

This work was supported by grants from the National Natural Science Foundation of China/Research Grants Council Joint Research Scheme (HKU 722/05 to M.L.F. and H.F.T.), the HKU Small Project Funding (201007176133 to K.M.N., C.W.S., and H.F.T.); Collaborative Research Fund of Hong Kong Research Grant Council (HKU 8/CRF/09 to C.W.S and H.F.T)

\section{Author Disclosure Statement}

The authors declare that no conflicting financial interests exist.

\section{References}

Ateghang, B., Wartenberg, M., Gassmann, M., et al. (2006). Regulation of cardiotrophin-1 expression in mouse embryonic stem cells by HIF-1\{alpha\} and intracellular reactive oxygen species. J. Cell Sci. 119, 1043-1052.

Au, K.W., Liao, S.Y., Lee, Y.K., et al. (2009). Effects of iron oxide nanoparticles on cardiac differentiation of embryonic stem cells. Biochem. Biophys. Res. Commun. 379, 898-903.

Bers, D.M. (2004). Macromolecular complexes regulating cardiac ryanodine receptor function. J. Mol. Cell Cardiol. 37, 417-429.

Bunn, H.F., and Poyton, R.O. (1996). Oxygen sensing and molecular adaptation to hypoxia. Physiol. Rev. 76, 839-885.

Chan, Y.C., Siu, C.W., Lau, Y.M., et al. (2009). Synergistic effects of inward rectifier (I) and pacemaker (I) currents on the induction of bioengineered cardiac automaticity. J. Cardiovasc. Electrophysiol. 20, 1048-1054.

Chen, H.F., Kuo, H.C., Chen, W., et al. (2009). A reduced oxygen tension $(5 \%)$ is not beneficial for maintaining human embryonic stem cells in the undifferentiated state with short splitting intervals. Hum. Reprod. 24, 71-80.

Chen, Y., Amende, I., Hampton, T.G., et al. (2006). Vascular endothelial growth factor promotes cardiomyocyte differentiation of embryonic stem cells. Am. J. Physiol. Heart Circ. Physiol. 291, H1653-H1658.

Ezashi, T., Das, P., and Roberts, R.M. (2005). Low $\mathrm{O}_{2}$ tensions and the prevention of differentiation of hES cells. Proc. Natl. Acad. Sci. USA 102, 4783-4788.

Fan, G.C., Yuan, Q., and Kranias, E.G. (2008). Regulatory roles of junctin in sarcoplasmic reticulum calcium cycling and myocardial function. Trends Cardiovasc. Med. 18, 1-5.
Fisher, S.A., and Burggren, W.W. (2007). Role of hypoxia in the evolution and development of the cardiovascular system. Antioxid. Redox Signal 9, 1339-1352.

Fu, J.D., Yu, H.M., Wang, R., et al. (2006). Developmental regulation of intracellular calcium transients during cardiomyocyte differentiation of mouse embryonic stem cells. Acta Pharmacol. Sin. 27, 901-910.

Graham, R.M., Frazier, D.P., Thompson, J.W., et al. (2004). A unique pathway of cardiac myocyte death caused by hypoxiaacidosis. J. Exp. Biol. 207, 3189-3200.

Huang, Y., Du, K.M., Xue, Z.H., et al. (2003). Cobalt chloride and low oxygen tension trigger differentiation of acute myeloid leukemic cells: possible mediation of hypoxia-inducible factor1alpha. Leukemia 17, 2065-2073.

Huang, Y., Hickey, R.P., Yeh, J.L., et al. (2004). Cardiac myocytespecific HIF-1alpha deletion alters vascularization, energy availability, calcium flux, and contractility in the normoxic heart. FASEB J. 18, 1138-1140.

Iyer, N.V., Kotch, L.E., Agani, F., et al. (1998). Cellular and developmental control of $\mathrm{O}_{2}$ homeostasis by hypoxia-inducible factor 1 alpha. Genes Dev. 12, 149-162.

Kaelin, W.G., Jr. (2002). How oxygen makes its presence felt. Genes Dev. 16, 1441-1445.

Kivirikko, K.I., and Myllyharju, J. (1998). Prolyl 4-hydroxylases and their protein disulfide isomerase subunit. Matrix Biol. 16, 357-368.

Knollmann, B.C. (2009). New roles of calsequestrin and triadin in cardiac muscle. J. Physiol. 587, 3081-3087.

Lieu, D.K., Liu, J., Siu, C.W., et al. (2009). Absence of transverse tubules contributes to non-uniform $\mathrm{Ca}(2+)$ wavefronts in mouse and human embryonic stem cell-derived cardiomyocytes. Stem Cells Dev. 18, 1493-1500.

Lin, S.K., Shun, C.T., Kok, S.H., et al. (2008). Hypoxia-stimulated vascular endothelial growth factor production in human nasal polyp fibroblasts: effect of epigallocatechin-3-gallate on hypoxia-inducible factor-1 alpha synthesis. Arch. Otolaryngol. Head Neck Surg. 134, 522-527.

Liu, J., Fu, J.D., Siu, C.W., et al. (2007). Functional sarcoplasmic reticulum for calcium handling of human embryonic stem cellderived cardiomyocytes: insights for driven maturation. Stem Cells 25, 3038-3044.

Liu, J., Lieu, D.K., Siu, C.W., et al. (2009). Facilitated maturation of $\mathrm{Ca} 2+$ handling properties of human embryonic stem cellderived cardiomyocytes by calsequestrin expression. Am. J. Physiol. Cell Physiol. 297, C152-C159.

Matsumoto, M., Makino, Y., Tanaka, T., et al. (2003). Induction of renoprotective gene expression by cobalt ameliorates ischemic injury of the kidney in rats. J. Am. Soc. Nephrol. 14, 1825-1832.

Moore, J.C., van Laake, L.W., Braam, S.R., et al. (2005). Human embryonic stem cells: genetic manipulation on the way to cardiac cell therapies. Reprod. Toxicol. 20, 377-391.

Moore, J.C., Fu, J., Chan, Y.C., et al. (2008). Distinct cardiogenic preferences of two human embryonic stem cell (hESC) lines are imprinted in their proteomes in the pluripotent state. Biochem. Biophys. Res. Commun. 372, 553-558.

Nagao, K., Taniyama, Y., Kietzmann, T., et al. (2008). HIF-1alpha signaling upstream of NKX2.5 is required for cardiac development in Xenopus. J. Biol. Chem. 283, 11841-11849.

Ng, K.M., Lee, Y.K., Chan, Y.C., et al. (2010). Exogenous expression of HIF-1 alpha promotes cardiac differentiation of embryonic stem cells. J. Mol. Cell Cardiol. 48, 1129-1137.

Ng, K.M., Lee, Y.K., Lai, W.H., et al. (2011). Exogenous expression of human apoA-I enhances cardiac differentiation of pluripotent stem cells. PLoS One 6, e19787. 
Niebruegge, S., Bauwens, C.L., Peerani, R., et al. (2009). Generation of human embryonic stem cell-derived mesoderm and cardiac cells using size-specified aggregates in an oxygencontrolled bioreactor. Biotechnol. Bioeng. 102, 493-507.

Ramirez-Bergeron, D.L., and Simon, M.C. (2001). Hypoxiainducible factor and the development of stem cells of the cardiovascular system. Stem Cells 19, 279-286.

Ramirez-Bergeron, D.L., Runge, A., Dahl, K.D.C., et al. (2004). Hypoxia affects mesoderm and enhances hemangioblast specification during early development. Development 131, 4623-4634.

Simon, M.C., and Keith, B. (2008). The role of oxygen availability in embryonic development and stem cell function. Nat. Rev. Mol. Cell Biol. 9, 285-296.

Siu, C.W., Moore, J.C., and Li, R.A. (2007). Human embryonic stem cell-derived cardiomyocytes for heart therapies. Cardiovasc. Hematol. Disord. Drug Targets 7, 145-152.

Wang, G.L., Jiang, B., Rue, E.A., and Semenza, G.L. (1995). Hypoxia-inducible factor 1 is a basic-helix-loop-helix-PAS heterodimer regulated by cellular $\mathrm{O}_{2}$ tension. Proc. Natl. Acad. Sci. USA 92, 5510-5514.
Xue, X., and Tomanek, R.J. (1999). Stimulation of coronary vasculogenesis/angiogenesis by hypoxia in cultured embryonic hearts. Dev Dynam. 216, 28-36.

Xinyun, C., Zhi, Z., Bin, Z., et al. (2010). Effects of cardiotrophin1 on differentiation and maturation of rat bone marrow mesenchymal stem cells induced with 5-azacytidine in vitro. Int. J. Cardiol. 143, 171-177.

Yang, L., Soonpaa, M.H., Adler, E.D., et al. (2008). Human cardiovascular progenitor cells develop from a KDR + embryonicstem-cell-derived population. Nature 453, 524-528.

Address correspondence to: Hung-Fat Tse, M.D., Ph.D. Cardiology Division Department of Medicine

The University of Hong Kong Rm. 1928, Block K, Queen Mary Hospital Hong Kong, Republic of China

E-mail: hftse@hkucc.hku.hk 
